\title{
Prenatal ultrasonographic diagnosis of cleft lip with or without cleft palate; pitfalls and considerations
}

\author{
Dong Wook Kim ${ }^{1,2+}$, Seung-Won Chung ${ }^{2,3 \dagger}$, Hwi-Dong Jung ${ }^{1}$ and Young-Soo Jung ${ }^{1 *}$
}

\begin{abstract}
Ultrasonographic examination is widely used for screening of abnormal findings on prenatal screening. Cleft lip with or without cleft palate of the fetus can also be screened by using ultrasonography. Presence of abnormal findings of the fetal lip or palate can be detected by the imaging professionals. However, such findings may not be familiar to oral and maxillofacial surgeons.

Oral and maxillofacial surgeons can use ultrasonographic imaging of fetal cleft lip with or without cleft palate to provide information regarding treatment protocols and outcomes to the parent. Therefore, surgeons should also be able to identify the abnormal details from the images, in order to setup proper treatment planning after the birth of the fetus.

We report two cases of cleft lip with or without cleft palate that the official readings of prenatal ultrasonography were inconsistent with the actual facial structure identified after birth. Also, critical and practical points in fetal ultrasonographic diagnosis are to be discussed.
\end{abstract}

Keywords: Cleft lip; Cleft palate; Prenatal ultrasonography

\section{Background}

The incidence of the various types of cleft lip (CL) with or without cleft palate $( \pm$ CP) is 1 per $700 \sim 1000$ live births worldwide [1,2]. Cleft lip with or without cleft palate $(\mathrm{CL} \pm \mathrm{CP})$ is the most common fetal craniofacial malformation that is screened during prenatal ultrasonographic examination [3].

An accurate prenatal diagnosis of the lip and palate anomaly is critical for establishing adequate long-term treatment planning, prediction of prognosis, and proper counseling with the parent [4]. Studies reporting the accuracy of $2 \mathrm{D}$ ultrasonography in detecting $\mathrm{CL} \pm \mathrm{CP}$ in low-risk populations demonstrate a wide variety in diagnostic accuracy [5]. The sensitivity of routine transabdominal ultrasonic scan at 20-weeks' gestation varies from $16 \%$ to $93 \%$, indicating a considerable proportion of misdiagnosis [5-8].

\footnotetext{
*Correspondence: ysjoms@yuhs.ac

${ }^{\dagger}$ Equal contributors

'Department of Oral and Maxillofacial Surgery, Oral Science Research Institute, Yonsei University College of Dentistry, 50-1 Yonsei-ro,

Seodaemun-gu, Seoul 120-752, Republic of Korea

Full list of author information is available at the end of the article
}

Meanwhile, health professionals are expected to have accurate and clear answers on possible questions from the parents following ultrasonographic examination $[4,5]$. Thus when the surgeons are to make treatment planning and counsel the parents, they should be aware of the potential pitfalls of the ultrasonography [4], as there can be possible inconsistencies between reading from the imaging professionals and the actual fetal facial structural anomalies.

Here we report two cases of CL with or without CP that the official readings of prenatal sonography and the actual facial structure identified after birth were somewhat inconsistent. After the case review, we would like to discuss the points to be considered in fetal ultrasonographic diagnosis.

\section{Case presentation \\ Case 1}

A 40-year-old healthy multipara was referred from local obstetrics and gynecology (OBGYN) clinic to the department of OBGYN in Severance hospital for further evaluation and management of congenital heart defect and

\section{黑 Springer}


unilateral CL $+\mathrm{CP}$ detected on ultrasonography at 26 weeks' gestation. The patient was referred to our department for prenatal counseling and treatment planning for the unilateral $\mathrm{CL}+\mathrm{CP}$ (Fig. 1).

After birth, the newborn was confirmed to have bilateral complete $\mathrm{CL}+\mathrm{CP}$, which was much more severe than that had been predicted (Fig. 2). Prenatally diagnosed congenital heart defect (double outlet right ventricle with ventricular septal defect) was also confirmed.

\section{Case 2}

A 29-year-old nullipara was under routine follow-up in the department of OBGYN in Severance hospital until 20 weeks' gestation. Until then, there were no known problems regarding the mother and the fetus, and the there were no detected anomalies in ultrasonography. From then, the mother was lost to follow-up. On 35 weeks' gestation, she revisited department of OBGYN in Severance hospital, referred from local OBGYN clinic due to cleft lip of the fetus detected on ultrasonography. Ultrasonographic examination in the department of OBGYN in our hospital revealed unilateral cleft lip (Fig. 3). After birth, the newborn was confirmed to have isolated incomplete CL (Fig. 4).

\section{Conclusion}

The Case 1 shows an example of actual anomaly confirmed after birth being severe than it was predicted based on the ultrasonography. In the case 2, cleft lip was detected relatively late in gestational period. We would like to discuss the factors affecting prenatal ultrasonographic diagnosis, and special considerations of cleft lip with or without palate regarding such cases.

\section{Accuracy of the prenatal ultrasonography}

The accuracy of sonography for prenatal diagnosis of $\mathrm{CL} \pm \mathrm{CP}$ is highly variable and dependent on the experience of the sonographer, maternal body habitus, gestational age, fetal position, and the amount of amniotic fluid and the type of clef $[4,9,10]$.

Cash et al. reported that when the overall detection rate for facial clefts was $65 \%$, the detection rate for CL with CP was $93 \%$, isolated CL was $67 \%$, and isolated $\mathrm{CP}$ was $22 \%$ [8]. Isolated cleft palate is reported to be rarely identified prenatally [6, 9]. Therefore, surgeons should to be aware that a negative ultrasound result does not necessarily mean that unborn child is without orofacial cleft. Transabdominal 2D ultrasonographic screening for orofacial clefts in a low-risk population has a relatively low detection rate and low false-positive diagnosis [5].

\section{Types of orofacial cleft}

It is important to differentiate the various types of orofacial clefts, because each type of orofacial cleft has different prognosis $[5,11]$. For instance, when a $C L$ is visualized on ultrasonography, it is difficult to determine whether the alveolus and secondary palate are involved [9]. When palate is involved, reconstruction technique, surgical implications would be more complicated, and the risk for chronic otitis media, hearing loss, abnormal

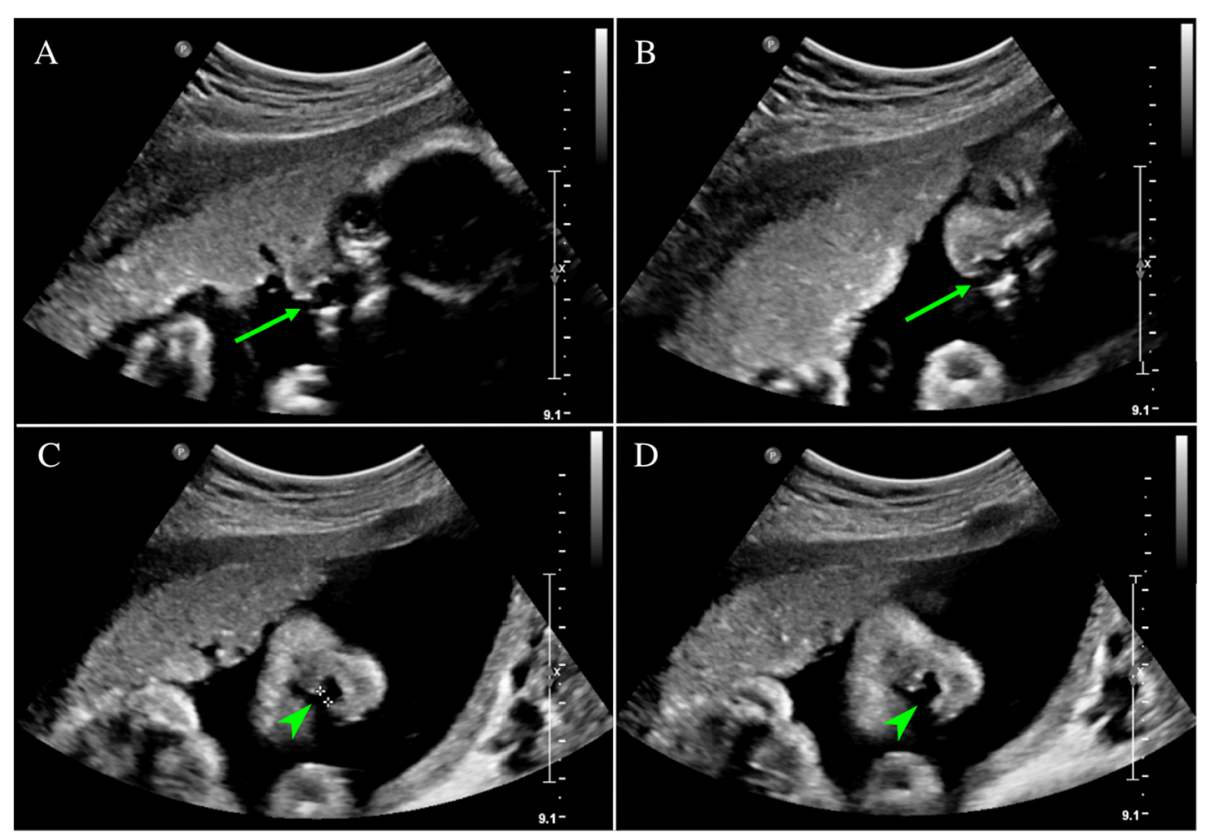

Fig. 1 Transabdominal ultrasonography of Case 1,26 weeks' gestation. a, b: Arrow indicates the cleft palate site. c, d: Arrowhead indicates the cleft lip site 


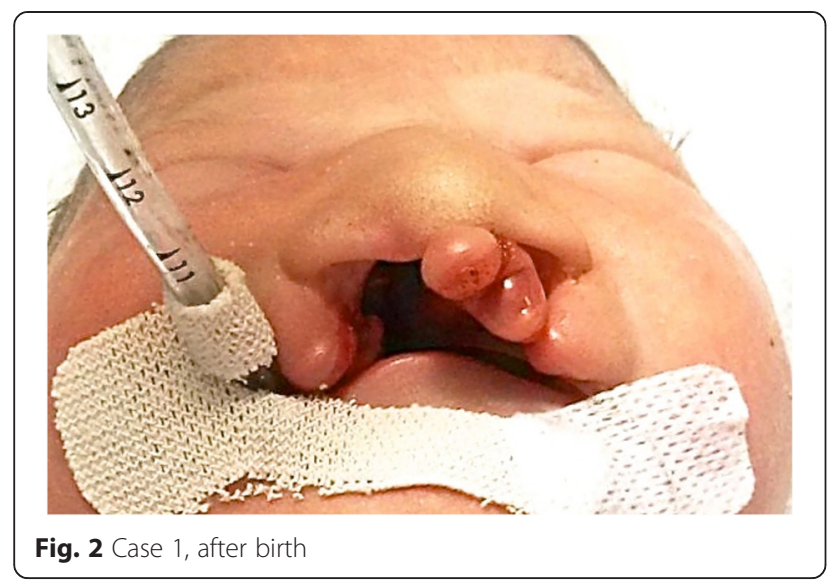

speech, and midfacial retrusion would increase [9]. This can be applied to the case 1 above, as the prenatal diagnosis was unilateral CL and it turned out to be bilateral complete CL with CP. Meanwhile, CL without CP is associated with a relatively favorable prognosis [11].

Unilateral incomplete CL can be subtle and undetectable until the third trimester [5]. While complete orofacial clefts can be detected in ultrasonographic examination as early as 16 weeks' gestation, unilateral incomplete CL is known to be detected after 27 weeks' gestation, as in Case $2[3,9]$. This minor labial clefting is not usually associated with other malformations and has relatively favorable prognosis $[9,11,12]$. At times, distinguishing between an incomplete and complete CL is difficult because there can be a thin band of tissue spanning the cleft even with a complete alveolar cleft $[9,13]$.

Even when a $\mathrm{CL}$ is visualized in ultrasonographic examination, it is difficult to determine whether the palate is also involved [9]. Approximately $90 \%$ of fetuses with a complete cleft of the primary palate will also have a complete cleft of the secondary palate [9]. Conversely, $10 \%$ of infants with complete unilateral or bilateral CL will have an intact secondary palate [9]. It is reported that reconstructed axial images obtained by 3D ultrasound of the fetal palate has high accuracy in identifying prenatal cleft palate when cleft lip is diagnosed at midtrimester 2D ultrasound screening [14].

\section{Advanced imaging modalities - 3D ultrasonography and MRI}

3D ultrasonography and prenatal MRI would improve the accuracy of prenatal diagnosis of orofacial clefts. 3D ultrasonography can provide more precise image of the defect and it has shown to enhance 2D examination significantly [5, 15-17]. While 3D ultrasonography can achieve a reliable diagnosis of fetal $\mathrm{CL} \pm \mathrm{CP}$, this does not rule out cases of CP only [5].

Magnetic resonance imaging (MRI) has been useful for prenatal diagnosis of fetal deformities compared with US, adding valuable information or supplying higher diagnostic accuracy [12]. The use of MRI is increasing

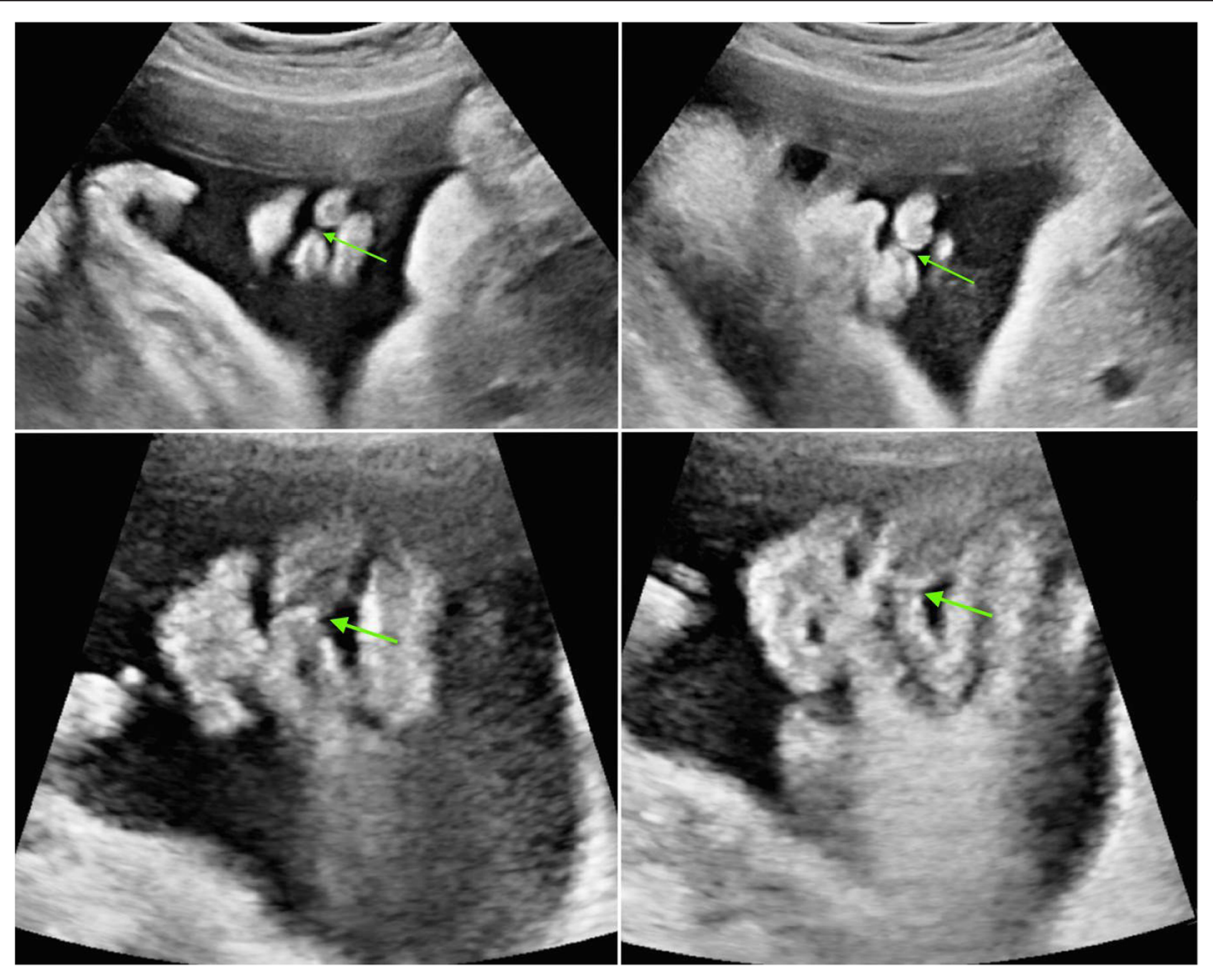

Fig. 3 Transabdominal ultrasonography of Case 2, 35 weeks' gestation. Arrows indicate cleft lip site 


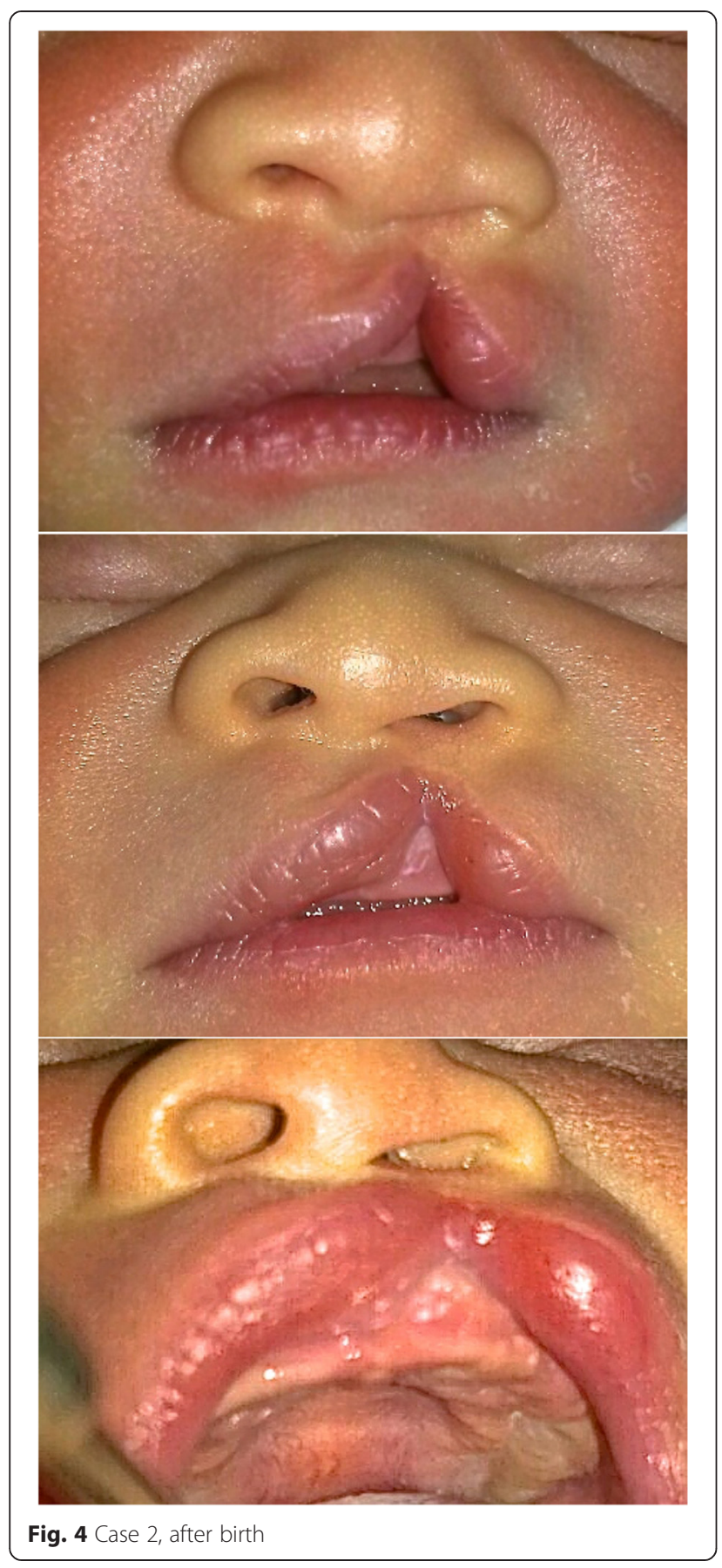

for evaluation of fetal abnormalities that are difficult to identify on ultrasonography alone [9]. Fetal MRI is less dependent than ultrasonography on optimal amniotic fluid volume, fetal position, and maternal body habitus [9]. Additionally, visualization of small structures on MRI is not limited by bone shadowing [9]. MRI enables visualization of the anterior six tooth buds (four of which arise from the premaxillary segment); and the continuous, smooth, echogenic, and horseshoe-shaped curve of the tooth-bearing alveolar ridge. This allows the diagnosis of alveolar cleft, and missing teeth buds, prenatally [9].

\section{Associated anomalies}

In the prenatal population, fetus with $\mathrm{CL}+\mathrm{CP}$ or cleft secondary palate usually has chromosomal abnormalities or other anomalies incompatible with survival [9]. Thus many fetuses with CL + CP die in utero or are spontaneously aborted and are never seen as newborns [3]. Therefore, the incidence of $\mathrm{CL} \pm \mathrm{CP}$ in the prenatal population is higher than that of postnatal population [9]. Thus prenatal ultrasonographic examination underscores the high incidence of spontaneous fetal loss that occurs whenever $\mathrm{CL} \pm \mathrm{CP}$ is associated with aneuploidy or other malformations [3].

Many syndromes have cleft as a part of their phenotypes and a cleft may be the only sign of a potential serious aneuploidy, as in above case 1 [18]. Severe additional anomalies can result in poor outcome, such as mortality [19]. Non-syndromic isolated CL \pm CP has low mortality and morbidity rates and are primarily a functional and esthetic problem [5]. CL only is associated with a very small percentage of chromosomal anomalies, as in Case 2 [18]. Ultrasonographic examination can never rule out a chromosomal aberration. Therefore, patients should receive genetic counseling and should be offered karyotypic analysis of their fetus, if needed [4].

\section{Counseling the parents}

Majority of the pregnant women and their partners are not expecting to identify a birth defect [4]. The initial shock caused by the discovery of a cleft is followed rapidly by fear, anger, sadness and guilt particularly if the baby is the first one [2,4]. Prenatal diagnosis poses many challenges for professionals involved in this process [4]. It is essential to emphasize to the parents-to-be that there is nothing they have done to account for the cleft, because feelings of guilt are very harsh [2]. However, when the diagnosis is known before delivery, the parents would have a chance to go through the grieving process [2].

Health professionals are expected to have accurate and clear answers on possible questions posed by the parents following ultrasonographic diagnosis $[4,5]$. The responsibility of the referral center is to define the nature of the structural defect with as much precision as possible. Furthermore, giving the information of burden of treatment and prognosis regarding a possible less-than-ideal outcome depending on the severity is critical to the process of counseling. Recurrence risk may also be one of the major concerns the parents and families may have to confront [4]. Families who have a previous child with $\mathrm{CL} \pm \mathrm{CP}$ have a 3 to 5 percent chance of recurrence, and if a parent and a previous child are both affected, the recurrence rate may be as high as 15 percent [3]. 


\section{Summary}

Prenatal diagnosis of orofacial cleft gives the parents the possibility to prepare themselves in an emotional and practical way [20]. A frank discussion of the cost of care and strategies for obtaining coverage for care can also relieve unspoken anxiety [4]. Knowing the diagnosis before delivery also allows the cleft team to discuss the plans, for example, feeding issues, type and geographical location of the cleft [2]. This would make delivery much less traumatic, especially for the parents and the extended family [2]. Our department offers information pamphlet for the future parents when they visit for first counseling. Information pamphlet should contain the concerns of parents-to-be, including longitudinal management of clefts and contact numbers [2].

For an effective counseling, accuracy of diagnosis is essential. Diagnosing techniques have been dramatically improved throughout the decades by technology, equipment and skills. However, health professionals must be aware of the possible pitfalls and related considerations of the ultrasonographic diagnosis for better treatment planning and counseling.

\section{Consent}

Written informed consent was obtained from the parents of the patients for publication of this Case report and any accompanying images. A copy of the written consent is available for review by the Editor-in-Chief of this journal.

\section{Abbreviations}

CL: Cleft lip; CP: Cleft palate; CL + CP: Cleft lip with cleft palate; CL $\pm C P$ : Cleft lip with or without cleft palate.

\section{Competing interests}

The authors declare that they have no competing interests.

\section{Authors' contributions}

DWK have made substantial contributions in reviewing articles, interpreting the data, and drafting the manuscript. SWC have made substantial contributions in revising the manuscript critically for important intellectual content. HDJ have made substantial contributions to conception and design. YSJ conceived of the study, and participated in its design and coordination and have given final approval of the version to be published. All authors read and approved the final manuscript.

\section{Acknowledgement}

This study was supported by a grant of the Korea Healthcare technology R\&D Project, Ministry for Health, Welfare \& Family Affairs, Republic of Korea (HI12C0297 (A120349)).

\footnotetext{
Author details

'Department of Oral and Maxillofacial Surgery, Oral Science Research Institute, Yonsei University College of Dentistry, 50-1 Yonsei-ro, Seodaemun-gu, Seoul 120-752, Republic of Korea. ${ }^{2}$ Graduate School, Yonsei University College of Dentistry, 50-1 Yonsei-ro, Seodaemun-gu, Seoul 120-752, Republic of Korea. ${ }^{3}$ Department of Dentistry, CHA Bundang Medical Center, CHA University, 59 Yatap-ro, Bundang-gu, Seongnam-si 463-712, Gyeonggi-do, Republic of Korea.
}

Received: 26 June 2015 Accepted: 14 July 2015

Published online: 14 August 2015

\section{References}

1. IPDTOC Working Group (2011) Prevalence at birth of cleft lip with or without cleft palate: data from the International Perinatal Database of Typical Oral Clefts (IPDTOC). Cleft Palate Craniofac J 48:66-81. doi:10.1597/09-217

2. Moss A (2001) Controversies in cleft lip and palate management. Ultrasound Obstet Gynecol 18:420-421. doi:10.1046/j.0960-7692.2001.00577.x

3. Benacerraf BR, Mulliken JB (1993) Fetal cleft lip and palate: sonographic diagnosis and postnatal outcome. Plast Reconstr Surg 92:1045

4. Jones MC (2002) Prenatal diagnosis of cleft lip and palate: detection rates, accuracy of ultrasonography, associated anomalies, and strategies for counseling. Cleft Palate Craniofac J 39:169-173, http://dx.doi.org/10.1597/ 1545-1569(2002)039<0169:PDOCLA>2.0.CO;2

5. Maarse W, Berge SJ, Pistorius L, van Barneveld T, Kon M, Breugem C, Mink van der Molen AB (2010) Diagnostic accuracy of transabdominal ultrasound in detecting prenatal cleft lip and palate: a systematic review. Ultrasound Obstet Gynecol 35:495-502. doi:10.1002/uog.7472

6. Shaikh D, Mercer NS, Sohan K, Kyle P, Soothill P (2001) Prenatal diagnosis of cleft lip and palate. Br J Plast Surg 54:288-289. doi:10.1054/bjps.2001.3575

7. Wayne C, Cook K, Sairam S, Hollis B, Thilaganathan B (2014) Sensitivity and accuracy of routine antenatal ultrasound screening for isolated facial clefts. Br J Radiol 75:584-589. doi:10.1259/bjr.75.895.750584

8. Cash C, Set P, Coleman N (2001) The accuracy of antenatal ultrasound in the detection of facial clefts in a low-risk screening population. Ultrasound Obstet Gynecol 18:432-436. doi:10.1046/j.0960-7692.2001.00508.x

9. Stroustrup Smith A, Estroff JA, Barnewolt CE, Mulliken JB, Levine D (2004) Prenatal diagnosis of cleft lip and cleft palate using MRI. AJR Am J Roentgenol 183:229-235. doi:10.2214/ajr.183.1.1830229

10. Evans MI, Hume RF Jr, Johnson MP, Treadwell MC, Krivchenia EL, Zador IE, Sokol RJ (1996) Integration of genetics and ultrasonography in prenatal diagnosis: just looking is not enough. Am J Obstet Gynecol 174:1925-1933. doi:10.1016/S0002-9378(96)70230-2

11. Nyberg DA, Sickler GK, Hegge FN, Kramer DJ, Kropp RJ (1995) Fetal cleft lip with and without cleft palate: US classification and correlation with outcome. Radiology 195:677-684. doi:10.1148/radiology.195.3.7753993

12. Wang G, Shan R, Zhao L, Zhu X, Zhang X (2011) Fetal cleft lip with and without cleft palate: comparison between MR imaging and US for prenatal diagnosis. Eur J Radiol 79:437-442. doi:10.1016/j.ejrad.2010.03.026

13. Mulliken JB, Benacerraf BR (2001) Prenatal diagnosis of cleft lip: what the sonologist needs to tell the surgeon. J Ultrasound Med 20:1159-1164

14. Bäumler M, Faure JM, Bigorre M, Bäumler Patris C, Boulot P, Demattei C, Captier G (2011) Accuracy of prenatal three-dimensional ultrasound in the diagnosis of cleft hard palate when cleft lip is present. Ultrasound Obstet Gynecol 38:440-444. doi:10.1002/uog.8933

15. Wang LM, Leung KY, Tang M (2007) Prenatal evaluation of facial clefts by three-dimensional extended imaging. Prenat Diagn 27:722-729. doi:10.1002/ pd. 1766

16. Carlson DE (2000) The ultrasound evaluation of cleft lip and palate-a clear winner for 3D. Ultrasound Obstet Gynecol 16:299-301. doi:10.1046/j.14690705.2000.00323.x

17. Rotten D, Levaillant JM (2004) Two- and three-dimensional sonographic assessment of the fetal face. 2. Analysis of cleft lip, alveolus and palate. Ultrasound Obstet Gynecol 24:402-411. doi:10.1002/uog.1718

18. Stoll C, Alembik Y, Dott B, Roth M-P (2007) Associated malformations in patients with oral clefts. Am J Med Genet A 143A:2463-2465. doi:10.1002/ ajmg.a.31764

19. Berge SJ, Plath H, Van de Vondel PT, Appel T, Niederhagen B, Von Lindern JJ, Reich RH, Hansmann M. (2001) Fetal cleft lip and palate: sonographic diagnosis, chromosomal abnormalities, associated anomalies and postnatal outcome in 70 fetuses. Ultrasound Obstet Gynecol 18:422-431. doi:10.1046/ j.0960-7692.2001.00575.x

20. Nusbaum R, Grubs RE, Losee JE, Weidman C, Ford MD, Marazita ML (2008) A qualitative description of receiving a diagnosis of clefting in the prenatal or postnatal period. J Genet Couns 17:336-350. doi:10.1007/s10897-008-9152-5 